OPEN ACCESS

Edited by:

Sebastien Christian Carpentier,

Bioversity International, Belgium

Reviewed by:

Dalma G. Castillo,

Instituto de Investigaciones

Agropecuarias (INIA), Chile

Mathilde Causse,

Genetics and Improvement of Fruit

and Vegetables (INRA), France

*Correspondence:

Anyela V. Camargo

anyela.camargorodriguez@niab.com

$$
\begin{array}{r}
\text { Specialty section: } \\
\text { This article was submitted to } \\
\text { Plant Breeding, } \\
\text { a section of the journal } \\
\text { Frontiers in Plant Science }
\end{array}
$$

Received: 05 February 2018

Accepted: 07 June 2018

Published: 09 July 2018

Citation:

Camargo AV, Mackay I, Mott R, Han J,

Doonan JH, Askew K, Corke F,

Williams K and Bentley AR (2018)

Functional Mapping of Quantitative

Trait Loci (QTLs) Associated With

Plant Performance in a Wheat MAGIC

Mapping Population.

Front. Plant Sci. 9:887.

doi: $10.3389 / f p / s .2018 .00887$

\section{Functional Mapping of Quantitative Trait Loci (QTLs) Associated With Plant Performance in a Wheat MAGIC Mapping Population}

\author{
Anyela V. Camargo ${ }^{1 *}$, lan Mackay ${ }^{1}$, Richard Mott ${ }^{2}$, Jiwan Han ${ }^{3}$, John H. Doonan ${ }^{3}$, \\ Karen Askew $^{3}$, Fiona Corke ${ }^{3}$, Kevin Williams ${ }^{3}$ and Alison R. Bentley ${ }^{1}$ \\ ${ }^{1}$ The John Bingham Laboratory, National Institute of Agricultural Botany, Cambridge, United Kingdom, ${ }^{2}$ Division of \\ Bioscience, Genetics Institute, University College London, London, United Kingdom, ${ }^{3}$ National Plant Phenomics Centre, \\ Institute of Biological Environmental and Rural Sciences, Aberystwyth University, Aberystwyth, United Kingdom
}

In crop genetic studies, the mapping of longitudinal data describing the spatio-temporal nature of agronomic traits can elucidate the factors influencing their formation and development. Here, we combine the mapping power and precision of a MAGIC wheat population with robust computational methods to track the spatio- temporal dynamics of traits associated with wheat performance. NIAB MAGIC lines were phenotyped throughout their lifecycle under smart house conditions. Growth models were fitted to the data describing growth trajectories of plant area, height, water use and senescence and fitted parameters were mapped as quantitative traits. Trait data from single time points were also mapped to determine when and how markers became and ceased to be significant. Assessment of temporal dynamics allowed the identification of marker-trait associations and tracking of trait development against the genetic contribution of key markers. We establish a data-driven approach for understanding complex agronomic traits and accelerate research in plant breeding.

Keywords: wheat, senescence, data science, phenology, phenotyping, MAGIC

\section{INTRODUCTION}

In crop genetics, the formation of dynamic biological traits such as height, size and color are usually governed by multiple temporal and spatial factors. Their genetic variation can be attributed to the collective response of multiple small effects associated to those dynamic traits (Anderegg, 2015). Thus, some genes may control trait development at a given plant developmental stage, others may alter, or control rates of change, transitions between consecutive stages (Yang and $\mathrm{Xu}, 2007$ ). These changes can be due to different genes that turn on or off at various times. In other words, a dynamic trait is, in part, governed by genes whose effects change with time. When a trait is measured over many developmental stages, e.g., plant area, it reveals the dynamic expression of the underlying genes associated with the trait (Wu et al., 2011). These might reveal critical aspects of vulnerability and response to biotic and abiotic stresses, and thereby predict the effects of climate change on these traits (Soudzilovskaia et al., 2013). 
Up until recently, genetic mapping of traits such height, area or senescence was done on a single data point, representing the value of the trait at a given stage. This approach, although practical, overlooked many of the factors that defined the process of formation and development of the trait. When breeding for elite varieties, it is widely accepted that the timing of key developmental transitions is associated with crop performance. In wheat for example, the transition from vegetative to reproductive growth can have major effects on biomass accumulation and harvest index. Therefore, understanding the extent at which dynamics traits develop in time, an in space, may be useful in breeding for higher yield and stress adaptation.

Advances in phenotyping technology, as well as the development of methods for the analysis of longitudinal data have made possible the mapping of quantitative trait loci (QTLs) underlying the dynamics of a developmental trait. For example, Yang et al. (2006) fitted longitudinal data from four traits to a polynomial growth trajectory and used intervalmapping to map growth parameters to the genome. Yang and $\mathrm{Xu}$ (2007) also fitted growth trajectories to Legendre polynomials but used a Bayesian shrinkage model for multiple QTL mapping of the curve parameters. Polynomial models force growth to follow a smooth curve, potentially of great complexity. However, polynomial functions tend to make spurious predictions, their polynomial order is difficult to determine and their model parameters difficult to interpret (Paine et al., 2012; Xiong et al., 2011) used a general functional regression approach to fit mouse behavioral data. Li and Sillanpää (2013) used a Bayesian non-parametric multiple-loci procedure. The method uses the Bayesian P-splines with (non-parametric) B-spline bases to specify the form of the QTL trajectory and a random walk prior to determining the curve's degree of smoothness. Al-Tamimi et al. (2016) used cubic smoothing splines to estimate plant growth and transpiration in a rice population over 13 days. Although they fitted a growth model, they did not map the curve's fitted parameters but instead individual time ranges.

In this study we analyzed phenotypic profiles derived from the daily screening of a large, eight-founder "NIAB elite MAGIC" wheat population to evaluate the genetic factors underlying the temporal changes in key traits associated with wheat performance such as plant height and water use. Growth curve models were fitted to plant area, height, water use and senescence over time and the fitted parameters of the growth trajectories were mapped to the wheat genome. Single time points were also individually mapped to the genomes and results from both analytic approaches compared. We demonstrate that both methods provide key insights that could not be captured otherwise. The growth curve approach identifies crucial markers through the crop growth time line and the single time point approach gives an indication of when and how those markers become and then cease to be significant.

\section{MATERIALS AND METHODS}

\section{Plant Material}

Phenotypes extracted from a subset of the NIAB Elite eightfounder MAGIC population described in Mackay et al.
(2014) were evaluated in this study. The complete population consists of approximately 1,000 recombinant inbred lines (RILs) generated from three cycles of recombination between eight elite United Kingdom wheat varieties followed by five rounds of selfing to derive RILs. More information about the population including complete pedigrees, genotypes and existing phenotyping data can be found at www.niab.com/MAGIC.

\section{Glasshouse Cultivation}

As described in Camargo et al. (2016), plants were assessed between mid-January 2015 and mid-April 2015 in a Smart house at The National Plant Phenomics Centre facilities in Aberystwyth, UK. MAGIC parents together with 208 RILs (Camargo et al., 2016) were sown on 20 Oct, 2014 under well-watered conditions, with two replicates per genotype. Two seeds were sown in $6 \mathrm{~cm}$ pots of Levington $\mathrm{F} 2$ compost. After germination [11 Oct, 2014, approximately 11 days after sowing (DAS)] the seedlings were thinned to one per pot and transferred to a controlled environment room for vernalization $\left(5^{\circ} \mathrm{C}, 16\right.$-h day length) for 9 weeks. Following vernalization plants were transplanted to 15 $\times 15 \times 20 \mathrm{~cm}$ pots of F2 compost and were transferred to the Smart house. Each pot was placed into a cart on a conveyor system to allow for automatic and regular phenotyping. Pots were weighed and watered automatically daily to $75 \%$ gravimetric soil water content. Growth conditions were set to 14 -h daylength using $600 \mathrm{~W}$ sodium lamps to supplement natural lighting. Temperature was set to $18^{\circ} \mathrm{C}$ day, and $15^{\circ} \mathrm{C}$ night.

\section{Phenotyping}

Daily imaging commenced when the plants were transferred to the conveyor belt following vernalization using a LemnaTec 3D Scanalyzer (LemnaTec, GmbH, Wuerselen, Germany) for image acquisition. Four RGB pictures $(2,056 \times 2,454$ pixels $)$ were taken of each plant, one top view and three side view with a $45^{\circ}$ horizontal rotation. All manually acquired and digitally derived traits and their abbreviations are provided in Table $\mathbf{1}$.

\section{Image Processing}

Digital images were processed using $\mathrm{C}++$ (software available at https:/github.com/NPPC-UK/PAT64V3_W8). Briefly, image color classification was used to separate plant from background and a binary image was produced where " 1 " corresponded to plant and "0" to anything else. Then, image features accounting for plant height, plant area and plant yellow area were calculated from the analysis of " 1 " area. Height was measured from the surface of the soil (the front edge of the pot) to the uppermost part of the plant. Area was the total amount of segmented area. Yellow area was the total amount of regions of yellow color in the segmented area and was used as a proxy for Senescence (Figure 1).

\section{Statistical and Quantitative Trait Locus Analysis}

Data analytics was performed using the R environment. QTL mapping was performed using the R package "HAPPY" for the analysis of multi-parental populations (Mott et al., 2000). SNP genotypes were used to infer ancestral haplotype mosaics for 
TABLE 1 | Plant trait descriptions.

\begin{tabular}{llll}
\hline $\begin{array}{l}\text { Abbreviation } \\
\text { in paper }\end{array}$ & Trait & Trait description & Unit \\
\hline Area & Area & Plant area in $\mathrm{mm}^{2}$ & $\mathrm{~mm}^{2}$ \\
Height & Height & Plant height & $\mathrm{mm}^{2}$ \\
TYP.Area & Senescence & Total yellow area (senescence) & $\mathrm{mm}^{2}$ \\
water_amount & Water Use & Water amount & $\mathrm{ml}$ \\
A & A & Minimum asymptote (Equation 2) & \\
C & C & x value at the inflection point of & \\
$B$ & the curve (Equation 2) & \\
$D$ & $\mathrm{D}$ & Slope factor (Equation 2) & \\
$\mu$ & $\mathrm{D}$ & Maximum value that can be & \\
$\lambda$ & $\mu$ & obtained (Equation 2) & \\
$\sigma$ & $\lambda$ & Curve's mean (Equation 12) & \\
$S$ & $\sigma$ & Curve's amplitude (Equation 12) & \\
& $S$ & Curve's standard deviation & \\
& & (Equation 12) & \\
& & Bimodal separation (Equation 12) &
\end{tabular}

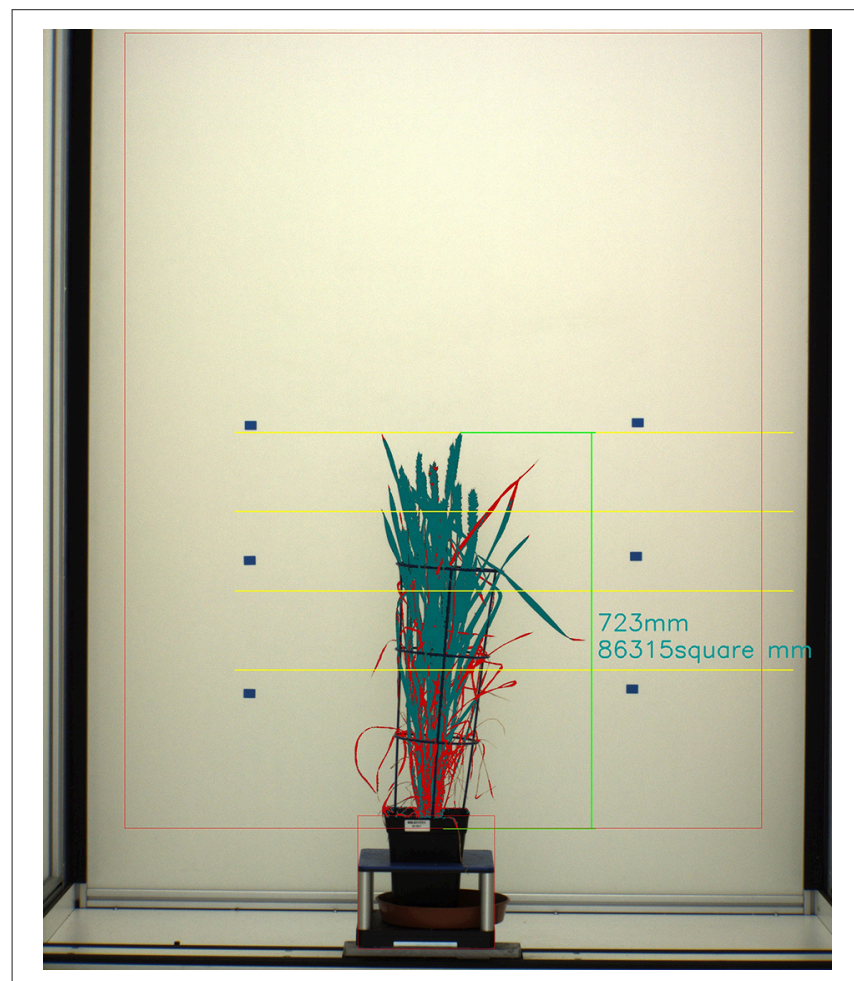

FIGURE 1 | Segmentation of image showing a wheat plant. Plant Area is represented in dark green and red. Plant Senescence is represented by red. Height is represented by vertical green line.

each MAGIC line and Single Marker analysis of variance was used to identify marker-trait associations. At each marker, lines were grouped according to their genotype and one-way ANOVA was used to test for significant differences between the groups. Marker-QTL association was indicated by F-statistics.
Significance for a given trait was tested by a permutation test (Camargo et al., 2008). First, the test statistic is calculated on the original data set. Next, phenotypes between lines are randomly shuffled 1,000 (B) times and test statistics are calculated on each shuffled data set. Finally, significance is estimated as the number of times $(K)$ the test statistic value obtained in the original data set was smaller than those of the shuffled data sets, and dividing that value by the number of random shuffles, i.e., K/B. We used $\max (-\log \mathrm{P})>4$ and $P<0.05$ as a cut-off value in the multiple QTL analysis to test for association (Storey and Tibshirani, 2003).

\section{Data Processing}

Data extracted from digital images were subject to preprocessing. First, time points were discarded if the whole set of RILs and parents were not imaged. Second, Cook's distance $(>4 \mu)$ was used to identify and remove outliers.

\section{Genetic Model for Longitudinal Traits}

A trait is called time-dependent, or longitudinal, if measured over time. Time-dependent traits can be analyzed by; (1) using a repeated measurements framework; (2) treating phenotypes measured across time as different traits or; (3) fitting growth curves to the phenotypic values measured over time then analyzing the parameters describing growth's trajectory (Yang et al., 2006). This study fitted growth curves because they reduced the dimensionality of the data and treated phenotypes measured across time as different traits without dismissing the correlation between them.

Many of the complexities of plant growth are commonly represented using non-linear growth models. Growth rates calculated this way can capture age- and size-dependent growth (Paine et al., 2012). To identify the growth model that best fitted our data, we fitted logistic (Equation 1), 4-parameter logistic (Equation 2) and Gompertz (Equation 3) models to the average of area, height, senescence and water use. In addition, mixture distributions were fitted using finite mixture models to estimate the parameters that best described the modes (Equations 4-7). The best growth model was selected by comparing coefficient of determinations (Equation 8) from each model.

The equations for each growth model are as follows:

\section{Logistic Model}

$$
f(t)=\frac{L}{\left(1+e^{-k\left(t-t_{0}\right)}\right)}
$$

where $L$ is the upper asymptote, $k$ is the growth rate and $t_{0}$ is the value of $t$ of the sigmoid curve's midpoint.

\section{4-Parameter Logistic Model}

$$
f(t)=D+\frac{(A-D)}{1+\left(\frac{t}{C}\right)^{B}}
$$

$A$ and $D$ are the bottom and top asymptotes (or the minimum and maximum values), $C$ is the inflection point (or the point on the $S$ shaped curve halfway between $A$ and $D$ ), and $B$ is the hill's slope, or the steepness of the curve. It could either be positive or negative. 


\section{Gompertz Model}

$$
f(t)=a e^{-b e^{-c t}}
$$

where, $a$ is an asymptote, $\left(\lim _{t \rightarrow \infty} a e^{-b e^{-c t}}=a e^{0}=a\right), b, c$ are positive numbers $b$ sets the displacement along the time axis $t$ and $c$ sets the growth rate (y scaling).

\section{Finite Mixture Model}

A finite mixture model (FMM) (Hathaway, 1985) is a probabilistic model for representing the presence of subpopulations (e.g., bi-modal distributions) within an overall population. In an FMM, the observed responses $y$ are assumed to come from $m$ distinct classes $f_{1}, f_{2}, \ldots, f m$ in proportions $\pi_{1}, \pi_{2}, \ldots$, $\pi_{g}$. In its simplest form, the density of a m-component mixture model as:

$$
h(y \mid x, \psi)=\sum_{i=1}^{m} \pi_{m} f\left(y \mid x, \theta_{i}\right)
$$

where $y$ is a dependent variable with conditional density $h, x$ is a vector of independent variables, $\pi_{i}$ is the prior probability of component $k, \theta_{i}$ is the component specific parameter vector for the density function $f$, and $\psi=\left(\pi_{i}, \ldots, \pi_{i}, \theta_{m}^{\prime}, \ldots, \theta_{m}^{\prime}\right)$ is the vector of all parameters (Hathaway, 1985).

The posterior probability that observation $(x, y)$ belongs to class $\mathrm{m}$ is given by:

$$
P(m \mid x, y, \psi)=\frac{\pi_{m} f\left(y \mid x, \theta_{m}\right)}{\sum_{k} \pi_{k} f\left(y \mid x, \theta_{k}\right)}
$$

The log-likelihood of a sample of $\mathrm{N}$ observations $\left\{\left(x_{1}, y_{1}\right), \ldots,\left(x_{N}, y_{N}\right)\right\}$ is given by:

$$
\log \mathrm{L}=\sum_{n=1}^{N} \operatorname{logh}\left(y_{n} \mid x_{n}, \psi\right)=\sum_{n=1}^{N} \log \left(\sum_{i=1}^{m} \pi_{i} f\left(y_{n} \mid x_{n}, \theta_{i}\right)\right.
$$

FMM uses the Expectation-maximization (EM) algorithm to refine starting values before maximum likelihood estimation via two steps:

Estimate-step: estimate the posterior class probabilities for each observation:

$$
\hat{p}_{n k}=P\left(k \mid x_{n}, y_{n}, \hat{\psi}\right)
$$

Using Equation (2) and derive the prior class probabilities as

$$
\hat{\pi}_{k}=\frac{1}{N} \sum_{n=1}^{N} \hat{p}_{n k}
$$

Maximize-step: maximize the log-likelihood for each component separately using the posterior probabilities as weights:

$$
\max \left(\theta_{k}\right) \sum_{n=1}^{N} \hat{p}_{n k} \log f\left(y_{n} \mid x_{n}, \theta_{k}\right)
$$

The E- and M-steps are repeated until either the likelihood improvement falls under a given threshold or a number of searches is reached.

Coefficient of determination $\left(R^{2}\right)$ is used for model selection:

$$
1-\frac{\sum_{i}\left(y_{i}-\hat{y}_{i}\right)^{2}}{\sum_{i}\left(y_{i}-\bar{y}_{i}\right)^{2}}
$$

\section{Heritability}

Growth curve parameters were estimated for each trait and for each plant, and parameters' heritability $\left(H^{2}\right)$ (Equation 9) and genetic $(g)$ and environmental $(e)$ covariances (Equation 10) and correlations (Equation 11) between parameters were estimated.

$$
H^{2}(x)=\frac{s_{g}^{2}(x)}{s_{g}^{2}(x)+s_{e}^{2}(x)}
$$

Where $H^{2}$ is the heritability for a given trait, $x$, and $s_{g}^{2}(x)$ and $s_{e}^{2}(x)$ are the genetic and environment variances for that trait.

$$
S(x, y)=\frac{s_{i}^{2}(x+y)-s_{i}^{2}(x)-s_{i}^{2}(y)}{2}
$$

Where $S$ is the covariance between two traits $(x, y), s^{2}$ is the variance and $i=g$ or $e$, genetic or environment

$$
r(x, y)=\frac{S(x+y)}{\sqrt[2]{s^{2}(x)+s^{2}(y)}}
$$

where $r$ is the correlation between two traits $(x, y)$.

\section{RESULTS}

\section{Trait Analysis}

Analysis of trait across time indicated that in general all the MAGIC lines and their parents maintained steady growth and water use up until 138 DAS (Figures 2A-C, blue dotted line) which coincided with the timing at which most of the plants reached GS55 ( 136 DAS) and started showing signs to senescence, as indicated in Camargo et al. (2016). Figure S1 shows the average pattern of leaf senescence plotted against the average of water consumption for all plants. The distribution of the curves suggests that the onset of senescence can be predicted from a plant's water consumption.

In addition, the time point at which the Water Use curve starts to rise for the second time, 170 DAS (Figure 2D, brown dotted line), also coincides with the time at which most plants initiate senescence at the Flag leaf (170 DAS) (Figure 2D).

\section{Growth Curve Analysis}

Area, Height and Senescence (TYP) showed a logistic growth (Figures S3-S9). Figures 2A-C plots average values of these three traits over time for founder lines, controls and the mean of the RILs. For the population mean, comparison of error sum of squares from the fitted values of several growth models indicated that these traits were best described by a 4-parameter logistic growth curve (Equation 2; Figures 3A-C). 


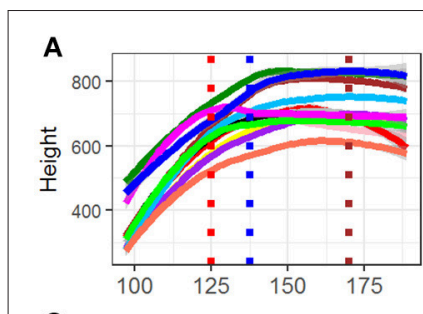

c
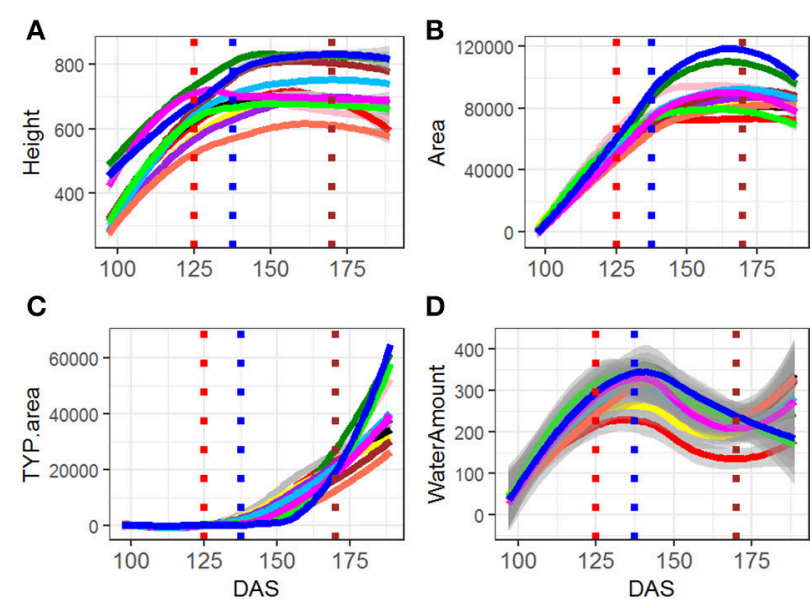

.
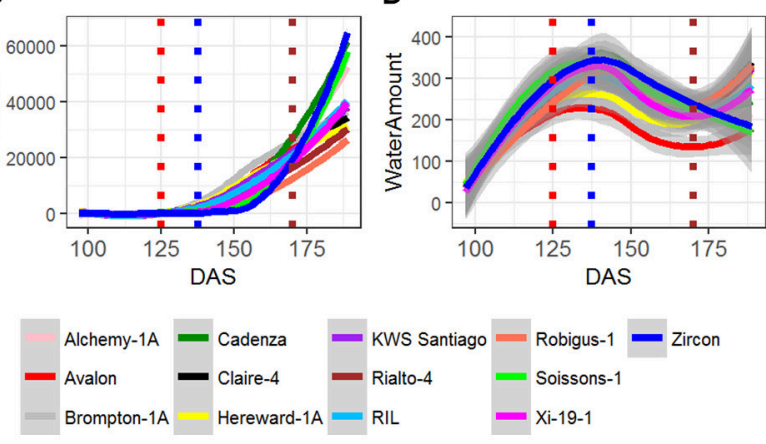

FIGURE 2 | Fitted curves for MAGIC parents and all RILS, for (A) Height, (B) Area, (C) TYP.Area Senescence, and (D) Water use. RIL is the average of all RILs per time point. For all the plants, red dotted line indicates average DAS for GS39, blue dotted line indicates average DAS for GS55 and brown dotted line indicates average DAS for FLS.

Growth parameters (Equation 2) were estimated for each trait and for each plant. Using data from the RILs, the heritability of these parameters and their genetic and environmental covariances and correlations were estimated. Results are shown in Tables S1-S6.

In the analysis of Area and Height growth curve parameters, $B$ (the curve's slope) has the highest heritability $(>0.8)$. The remaining parameters have heritability below 0.5 indicating that the rate at which a plant grows is highly heritable. However, $B$ did not show strong genetic or environmental correlations with the other parameters. $A$ and $C$ (the bottom asymptote and inflection point, respectively) were highly correlated both genetically and environmentally $(r>0.9)$ and $A$ and $D$ were negatively correlated $(<-0.6)$ at the genetic level. Senescence growth curve parameters did not show high heritability for any parameter. However, the genetic and environment correlations between $A$ and $D$ were negative $(<-0.6)$ and there was a large positive environment correlation (0.9) between $B$ and $D$.

In comparison to Area, Height and Senescence's curves, the Water Use curve was bi-modal (Figures 2A-C). The peak of the first mode was around 130 DAS and for the second around 180 DAS. Given the bi-modality, a mixture model (Equation 4) was fitted to the data to estimate the parameters describing the two modes; their amplitudes, $\lambda$, means, $\mu$, and standard deviations, $\sigma$ (Figure 3). The separation (Zhang et al., 2003) between the two modes was calculated as:

$$
S=\frac{\mu_{2}-\mu_{1}}{\left(2 \sigma_{1}+2 \sigma_{2}\right)}
$$

Growth parameters $\mu_{1}, \lambda_{1}$, and $\sigma_{1}$, corresponding to the first mode, $\mu_{2}$, and $\sigma_{2}$, corresponding to the second mode, were estimated for each trait and for each plant. Parameters for the RILs were then used to estimate heritabilities, co-variances and correlations. Results shown in Tables S7, S8 indicate that none of the growth parameters were highly heritable $\left(H^{2}<\right.$ 0.2 ). With such low heritabilities, genetic correlations are very poorly estimated and correlations between parameters are largely environmental. The pattern of these correlations indicates that early peak Water Use (smaller $\mu_{1}$ ) is associated with more water being used in the early period (high $\lambda_{1}$, and $\sigma_{1}$ ) and that this delays the second water uptake phase (high $\mu_{2}$ ). Xie et al. (2015) identified a similar Water Use peak after anthesis, and identified positive relationships between maximum Senescence rate and the time for maximum grain dimensions. We also compared thermal time against Water Use after anthesis and found a similar peak after that stage (Figure S2) which confirms the two peaks observed in the Water Use curve.

\section{Quantitative Trait Loci}

The lines were genotyped using the Illumina Infinium iSelect 80,000 SNP wheat array ("80 K array," http://www.illumina. com/), described in Yang et al. (2006). Prior to QTL mapping, a test for population structure was performed as indicated in Camargo et al. (2016).

\section{Analysing Individual Time Points}

Analysis of individual phenotypes at each time point identified loci associated with all traits but not with all the time points. For Area, seven significant QTLs were found on chr4D (Table S9), peak QTL corresponded to the marker RAC875_rep_c105718_585, between 11.0 and $23.0 \mathrm{Mb}$ (90\% confidence region) (Table 2, Figure 12). This QTL became highly significant at around 136 DAS and reached its maximum statistical significance at 166DAS (Figure 4).

For Height, the following QTLs were identified across different chromosomes: 13 were found on chr4D (Table S9), peak QTLs corresponded to the markers Kukri_rep_c68594_530 (10.0-21.0 Mb), RAC875_c6922_291 (10.0-13.0 Mb), RAC875_c1673_193 (15.0-21.0 Mb), RAC875_rep_c105718_585 $(11.0-23.0 \mathrm{Mb})$ and Tdurum_contig42083_1539 (10.0$20.0 \mathrm{Mb})$. Two were found on chr5A corresponding to wsnp_Ra_c44756_51084202 and wsnp_Ex_c113235_94249366. Two were found on chr5A corresponding to IAAV1650 (62.6$78.6 \mathrm{Mb})$ and BS00011360_51 (62.7-78.5 Mb). The statistical significance of the QTLs on chr5A was high $(>6.0 \log \mathrm{P})$ at early plant development ( $\sim 97 \mathrm{DAS})$ and decreased as the plant matured. The QTLs on chr5B showed a similar pattern but their statistical significance was low. In contrast, the statistical significance of the QTLs on chr4D was low $(<2.0 \log \mathrm{P})$ during early plant development ( $~ 97$ DAS) but became high around 136 DAS and reached their maximum significance level at 158 DAS which coincided with the time the average plant reached its maximum growth (Figure 5).

For Senescence, one QTL was found on chr4D, corresponding to the marker RAC875_c1673_193, and one on chr2D corresponding to Kukri_c27309_590.The statistical significance 

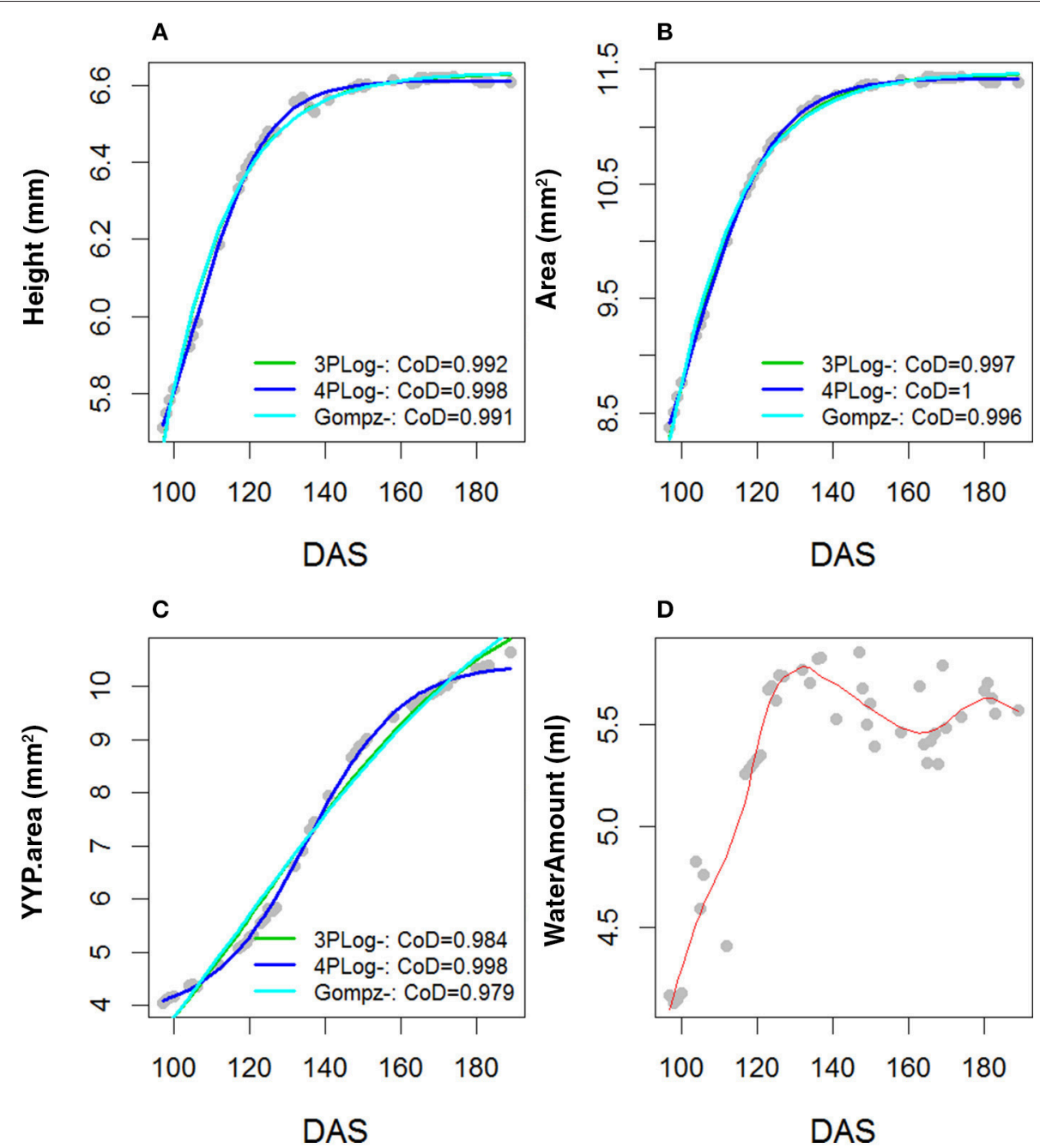

FIGURE 3 | Comparison of $R^{2}$ across three parameter logistic, four parameters logistic and Gompertz. Growth models were fitted to the population mean and for the traits (A) Height, (B) Area, (C) TYP. Area (Senescence) and (D) Water Amount. The bi-modal shape of the water amount curve was fitted with a Gaussian type growth model.

of the QTLs on chr4D was low $(<2.0 \log \mathrm{P})$ at early plant development ( $\sim 97$ DAS) but became high at around 148 DAS and reached their peak at 189 DAS, at the end of the plant's life (Figure 6).

For Water Use, one QTL was found on chr2D, corresponding to the marker GENE_0137_147 (12.5-13.7 Mb). Significance level of the QTL on chr2D was high at around 136 DAS and reached its maximum at 166DAS (Figure 7), after that significance levels became lower.

QTL associated with RAC875_rep_c105718_585 on chr4D were common for Area and Height and with RAC875_c1673_193 on chr4D for Height and Senescence. Both QTL became highly significant at around 136 DAS.

Figure 12 shows locations of peak QTLs located on chromosomes 2D, 4A, 5A, and 7B. Most peak QTLs are clustered within short distance except for Tdurum_contig42083_1539 on chr $4 \mathrm{~A}$ which is isolated for the main cluster.

\section{Analysing of Growth Curve Parameter}

Growth curve parameters fitted to Area across time identified nine QTLs associated with the parameter " $B$ " on chr4D (Table S9). The peak QTL was BS00022276_51 (Figure 8). The analysis of growth parameters for Height also identified 13 QTLs on chr4D. The peak QTL was RAC875_c1673_193 (Figure 9) which was also linked to Area and Height.

For Senescence growth parameters, one QTL on chr7B was associated to the " $A$ " parameter at marker BS00087197_51 (74.674.7) (Figure 10). For Water Use growth parameters, we did not identify QTLs (Figure 11).

\section{DISCUSSION}

This work aimed to understand the genetic elements influencing the temporal and spatial changes in key traits such as plant height and area, the timing of senescence and the 


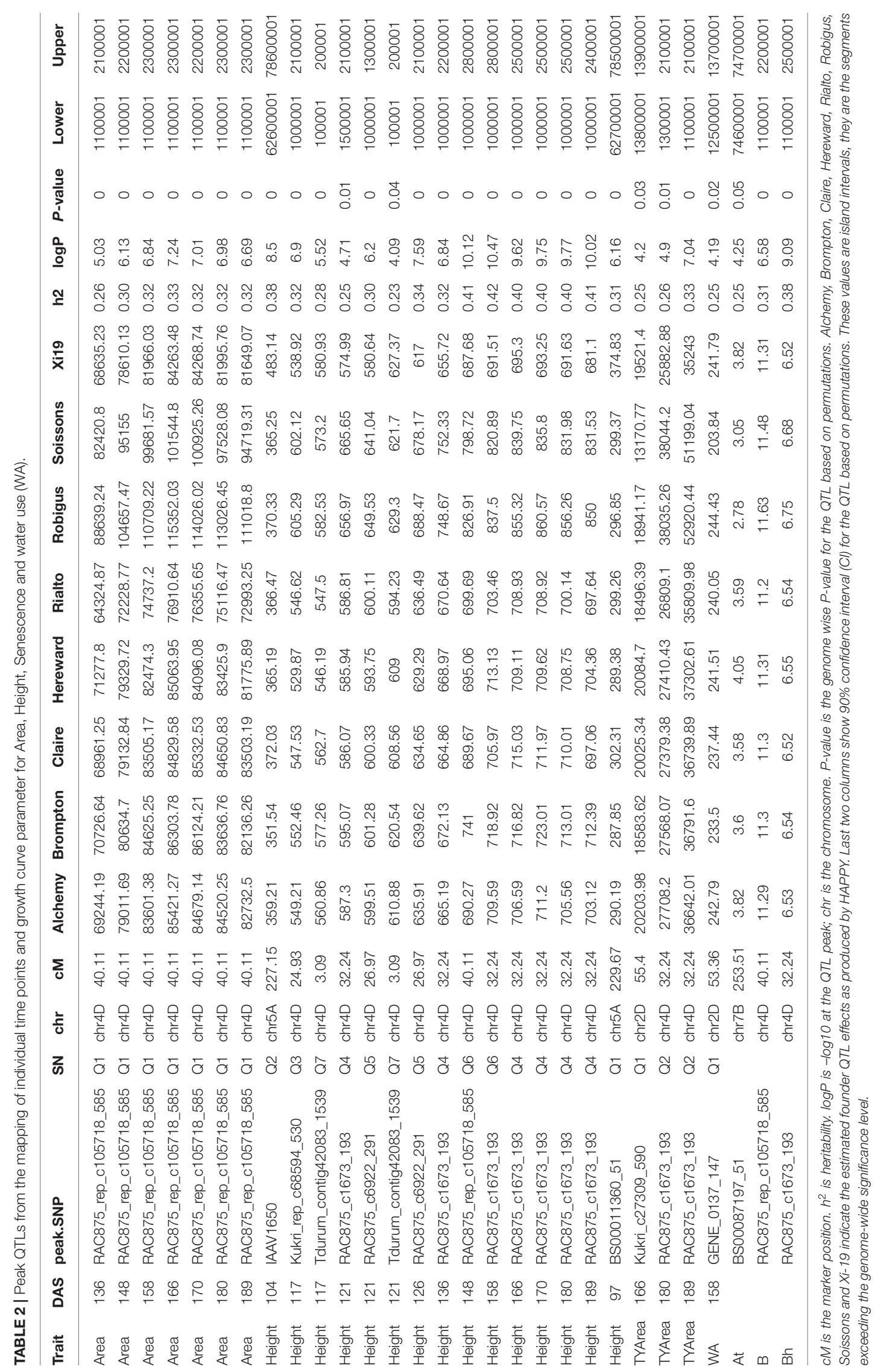




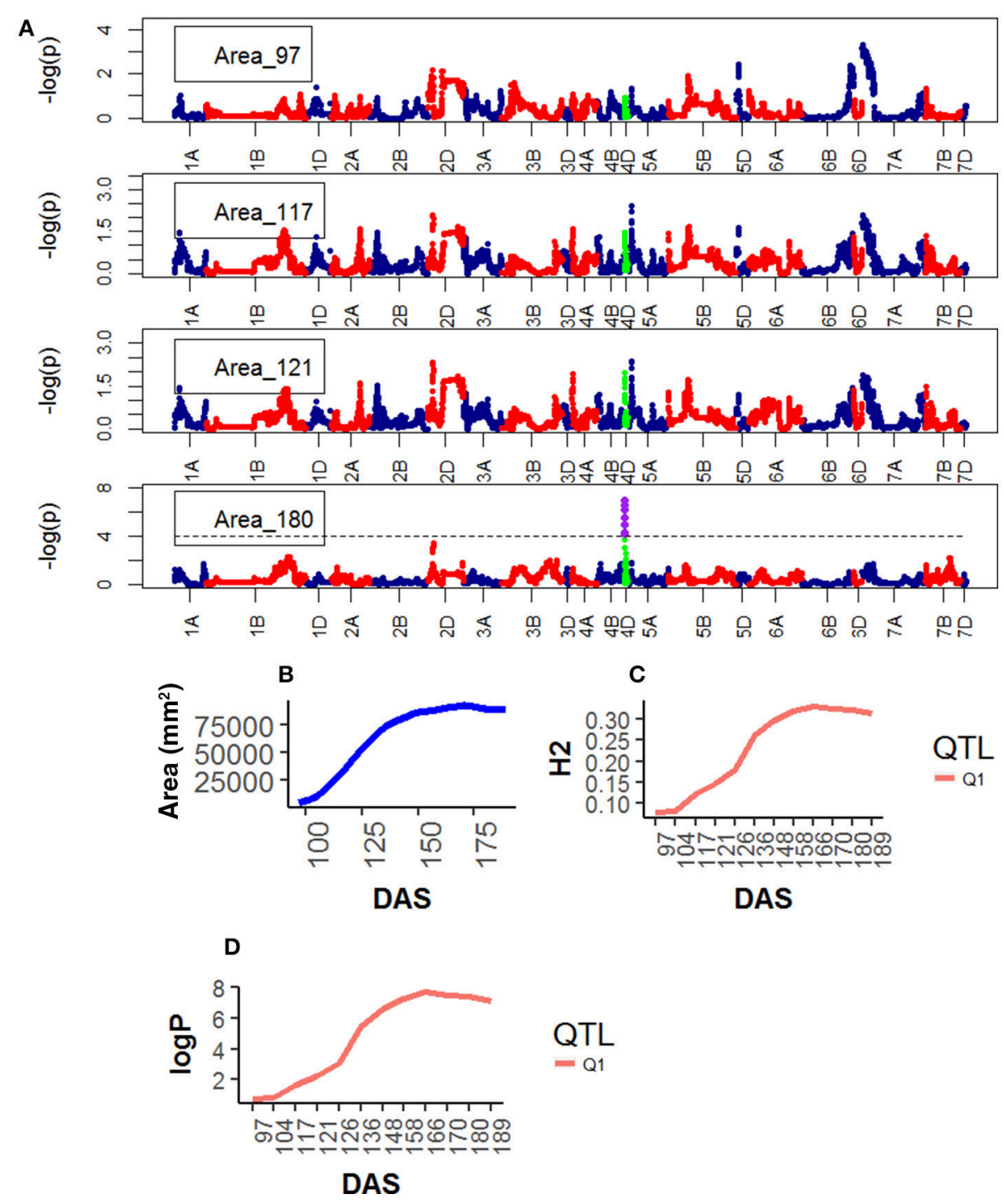

FIGURE 4 | Longitudinal analysis of the trait Area. (A) Manhattan plots highlighting chr4D at four DAS. Purple dots indicate QTLs with $P$-value $<0.05$ and max(-logP) > 4; (B) Population Area mean (C) heritability $\left(\mathrm{H}^{2}\right)$ for peak QLTs (Table 2), (D) -logP.

dynamics of water use. Since plant traits were measured across time, we used two different approaches to analyse growth trajectories: first, growth curves were fitted to the phenotypic values of each trait measured over time. Second, phenotypic measurements were treated as different traits and analyzed individually.

For the analysis of growth curves, we initially fitted second, third and fourth degree polynomials of the form $R G R=\mathrm{b}_{0}+$ $\mathrm{b}_{1} \mathrm{t}+\mathrm{b}_{2} \mathrm{t}^{2}+\ldots+\mathrm{b}_{n} t^{n}$ to the longitudinal data (Poorter, 1989), and performed QTL mapping over the polynomials' coefficients. Polynomials curves are easy to fit because they use standard linear modeling procedures and they can also approximate non-linear functions relatively well. However, results of QTL mapping did not identify any marker association from any of the polynomials fitted to the longitudinal data.
Higher order polynomials are difficult to interpret and are unlikely to have an obvious biological meaning: they describe the form of the curve rather than offering any natural explanation. In contrast, the logistic have been used successfully to model growth, and the parameters better describe the biology of growth (Paine et al., 2012). That QTL are found for the growth curve parameters but not for any of (or the higher order) polynomial regression coefficients is in agreement with this interpretation.

After evaluating curve fitting by using polynomials, we fitted non-linear plant growth models to the longitudinal data and through the comparison of $R^{2}$ identified the 4-parameter logistic model as the model that best fitted the phenotypic traits describing the growth trajectories of area, height and senescence. As explained later in this section, QTL mapping of curve parameters identified significant marker associations. 


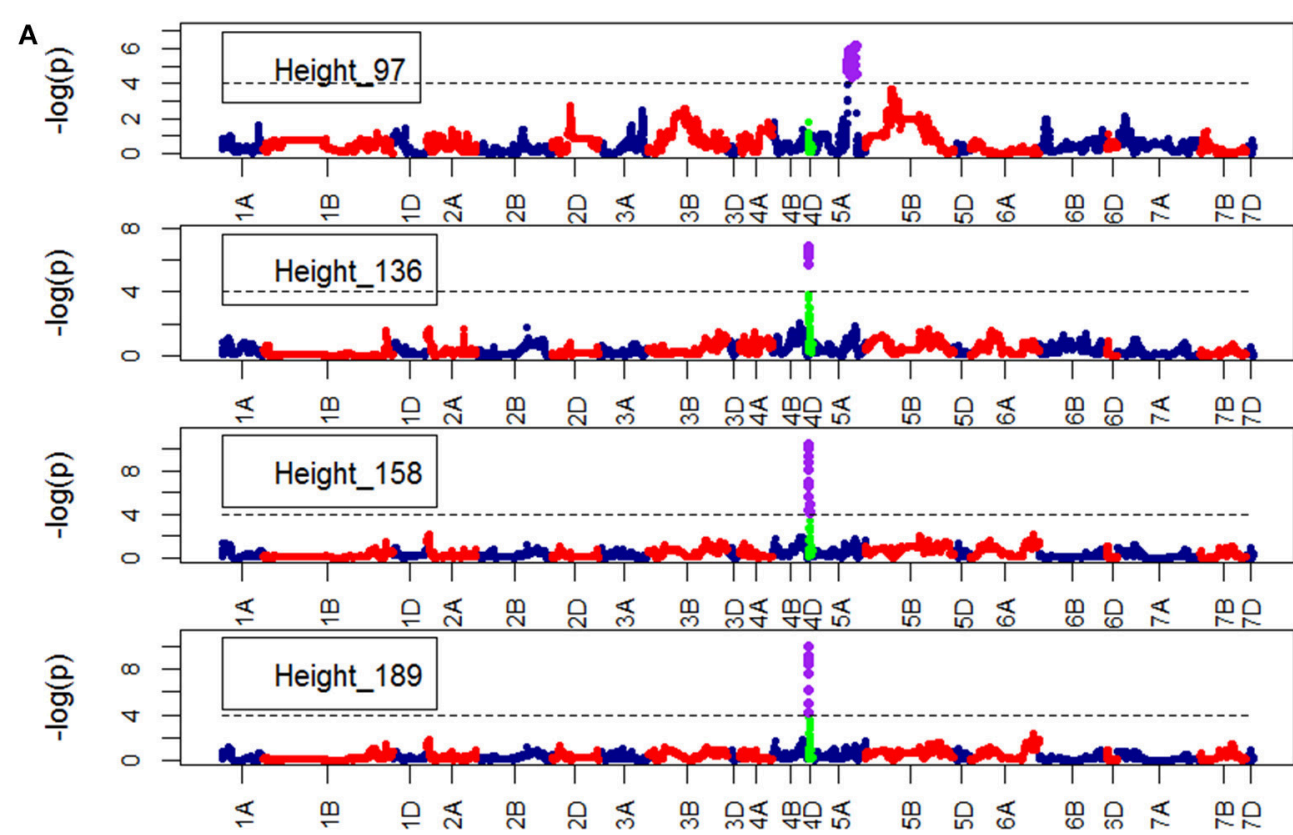

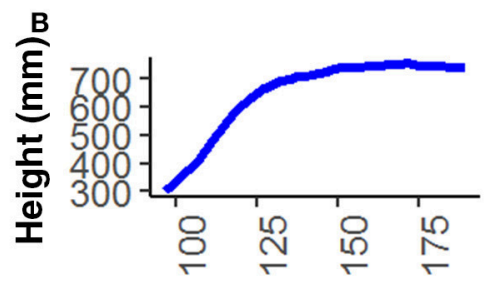

DAS

D

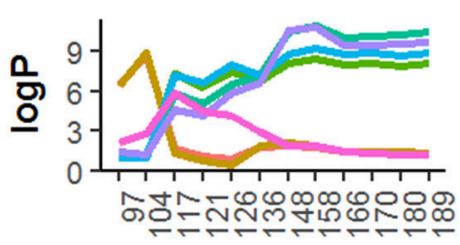

DAS
C

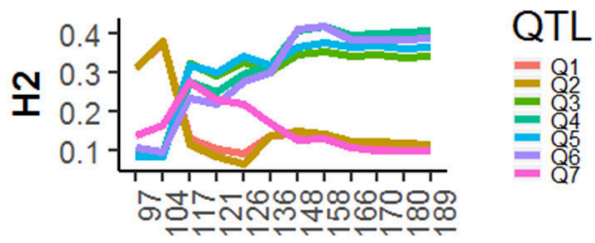

DAS

FIGURE 5 | Longitudinal analysis of the trait Height (A) Manhattan plots highlighting chr4D at four DAS. Purple dots indicate QTLs with $P$-value $<0.05$ and $\max (-\log P)>4$; (B) Population Area mean $(\mathbf{C})$ heritability $\left(\mathrm{H}^{2}\right)$ showing peak markers (Table 2) per chromosome $[P<0.05$, max $(-\log )$ ], (D) LOD scores [max $(-\log P)]$ showing peak markers per chromosome $[P<0.05$, max max $(-\log P)]$.

Comparison between growth curve parameters, identified curve steepness (" $B$ ") as the most heritable $(>0.8)$ for area and height but not for senescence. However, " $B$ " was not correlated to any other parameter in contrast to " $A$ " and " $C$," which were highly correlated both genetically and environmentally $(r>0.9)$, indicating that phenotypic outcome was highly dependent on genotype.

Since the Water Use curve showed a different growth trajectory (Figure 3D), having two modes, a multi-modal modeling approach was used to model the heterogeneity of the Water Use curve. When affecting fitness related traits such as survival or reproduction, individual heterogeneity plays a key role in population dynamics and life history evolution. However, it is only recently that properly accounting for individual heterogeneity when studying population dynamics has been made possible through the use of high-throughput technologies and the development of appropriate statistical models (Ford et al., 2015; Gimenez et al., 2017). In this study, we used a similar approach to Bresson et al. (2015) to model the bi-modality of the curve trajectory describing Water Use. Although, the analysis of growth parameters did not identify highly heritable parameters or high correlations between them, we realized the 


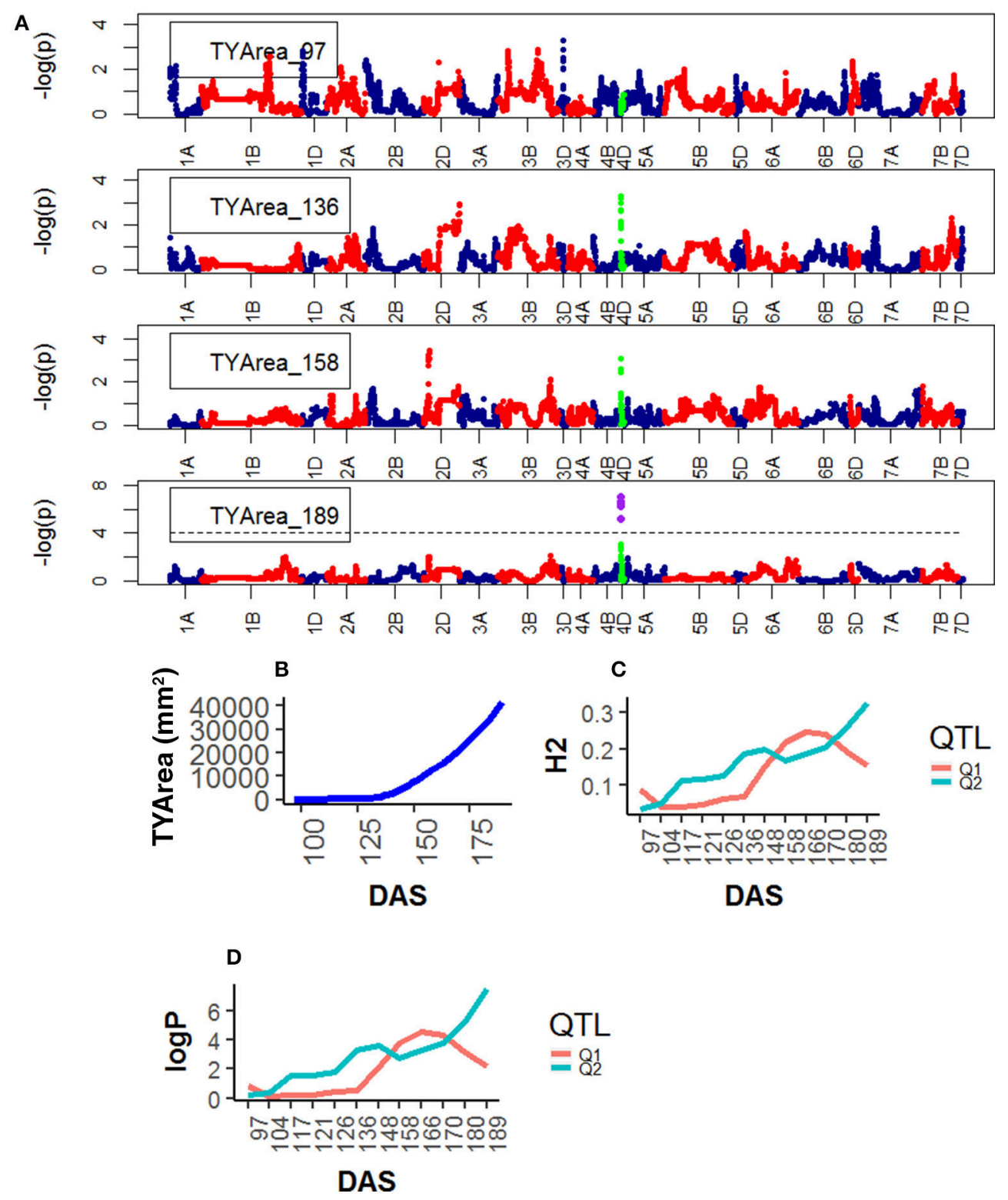

FIGURE 6 | Time resolved analyses for the trait Senescence (TYP) (A) Manhattan plots highlighting chr4D at four DAS. Purple dots indicate QTLs with $P$-value $<0.05$ and $\max (-\log P)>4$; (B) Population Area mean $(\mathbf{C})$ heritability $\left(H^{2}\right)$ showing peak markers (Table 2) per chromosome $[P<0.05, \max (-\log P)]$, (D) LOD scores $[\max (-\log P)]$ showing peak markers per chromosome $[P<0.05$, $\max (-\log P)]$

curve trajectory followed a similar pattern of observed by Xie et al. (2015) when they were looking into water accumulation and grain weight in wheat. The low heritability of Water Use growth parameters could also be explained by the sensitivity of water use to environmental variables such as changes in VPD.

Genetic mapping of individual phenotypic values measures across time not only highlighted key markers associated with area, height, senescence and water use but also indicated when marker effects became especially large or when their signal decayed. Most importantly, when comparing the plots on
Figure 2 and those on Figures 4-7B,C, one can assess how trait development correlates with relative QTL strength.

Genetic mapping analysis identified peak QTLs for the traits Senescence (Q1) and Water Use (Q1) on chr2D at the location of Ppd-D1, the photoperiod sensitivity (Bentley et al., 2013) locus. The effect was highly significant at around 148 DAS and became less significant after that. In addition to the mapping of phenological traits, we also looked for markers associated with morphological traits. For example, strong markers on chr4D such as RAC875_rep_c105718_585 and RAC875_rep_c105718_585 were linked with plant Area and plant Height. In both cases, 


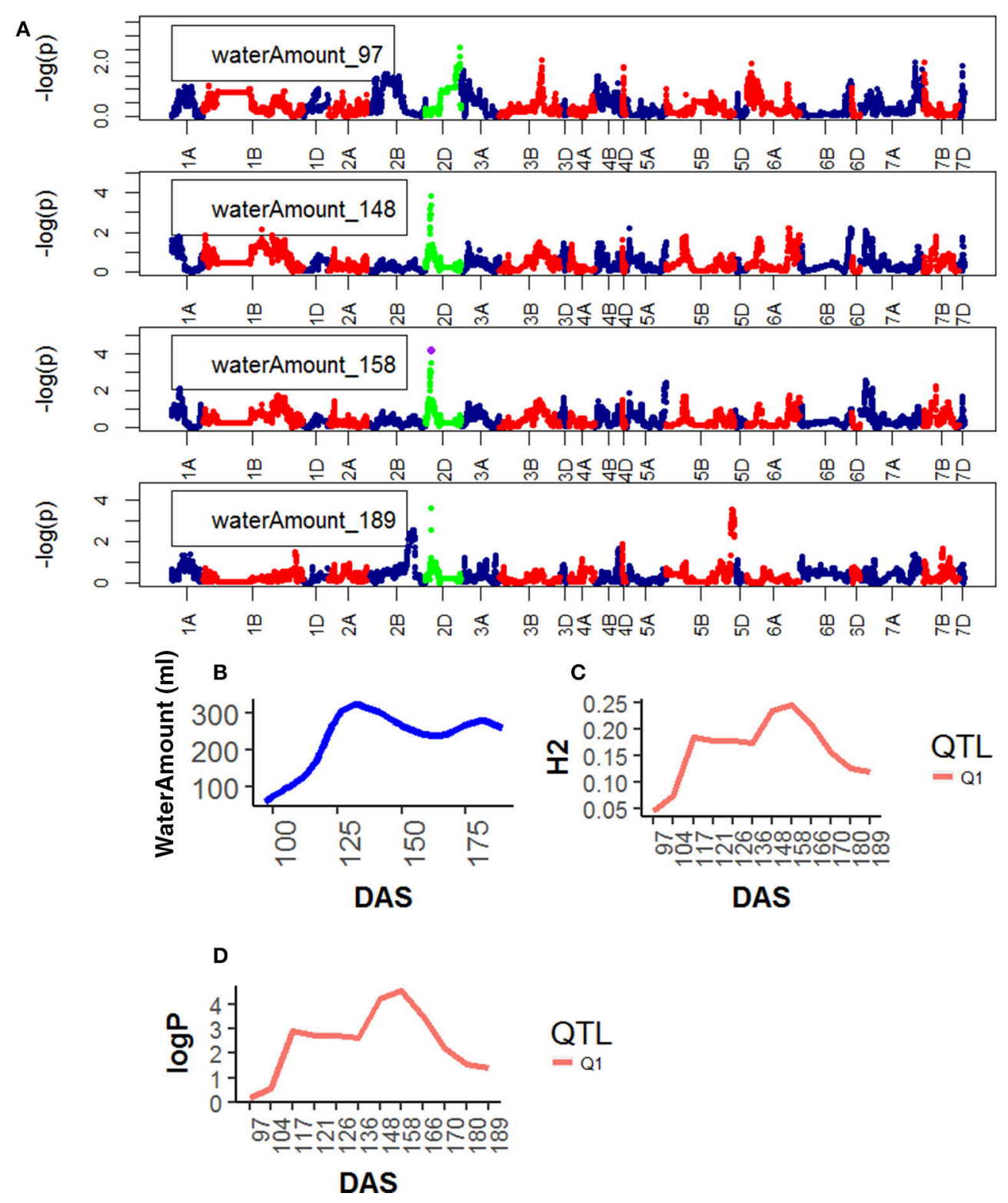

FIGURE 7 | Time resolved analyses for the trait Water use (Water amount) (A) Manhattan plots highlighting chr4D at four DAS. Purple dots indicate QTLs with P-value $<0.05$ and $\max (-\log P)>4$; (B) Population Area mean (C) heritability $\left(\mathrm{H}^{2}\right)$ showing peak markers (Table 2) per chromosome $[P<0.05$, max $(-\log P)]$, (D) LOD scores $(\log P)$ showing peak markers per chromosome $[P<0.05, \max (-\log P)]$.

the QTL interval includes the Rht-D1 (Rht2) locus. The Rht$D 1 b$ allele at this locus has been associated with a reduction in plant height and several other morphological traits and is segregating in the MAGIC population (the dwarfing allele at this locus is present in all parent lines except Robigus and Soissons, which carry the dwarfing allele Rht-B1b). Table 2 confirms that the estimated founder QTL effect contribution of Robigus and Soissons over height and for the RAC875_rep_c105718_585 marker is higher than for the other parents.

We also identified two strong effects $(-\log \mathrm{P}>8.00)$ on chr5A associated with plant Height at markers IAAV1650 and
BS00011360_51. We believe these markers are closely linked to the vernalization gene Vrn-A1 which plays an important role in the vernalization process in diploid Einkorn wheat (Triticum monococcum) and polyploid common wheat (Triticum aestivum; Kiss et al., 2014). Finally, a peak marker was found on chr7B which was associated to Senescence. Table 2 shows that the estimated founder QTL effect contribution Xi19 over Height and for the IAAV1650 and BS00011360_51 markers is higher than for the other parents. BS00011360_51 is likely to be the VRN-B3 gene which has been associated with increased yield in EU 2011 and GBR 2010 wheat trials (Bentley et al., 2014). 

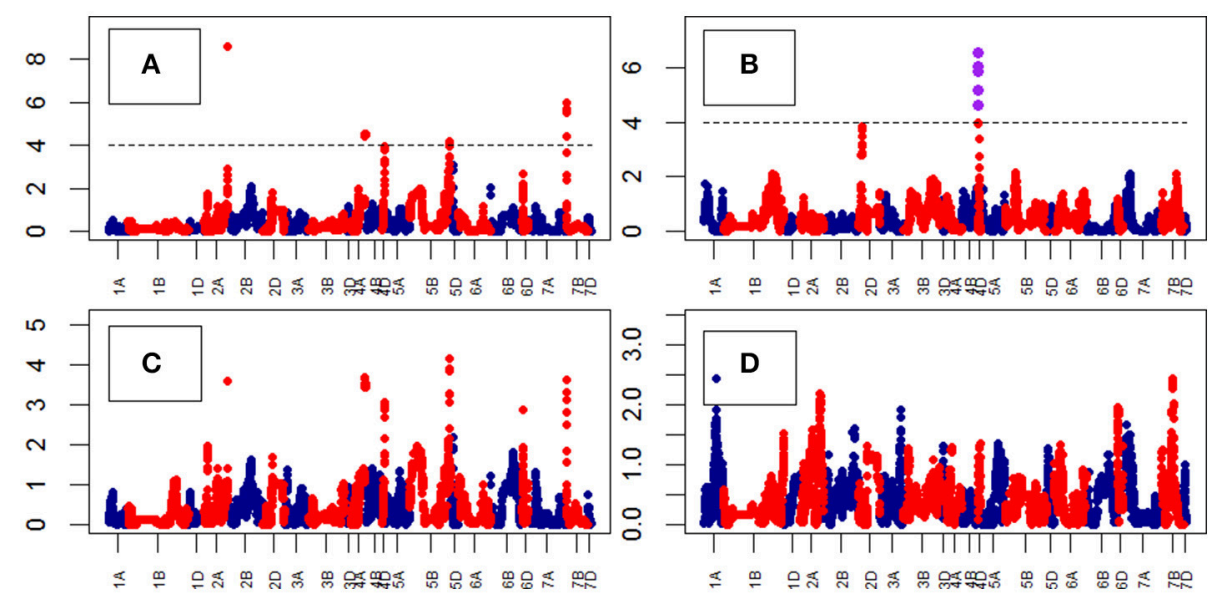

FIGURE 8 | Manhattan plots from the genetic mapping of the curve's growth parameters corresponding to the trait Area. Purple dots indicate QTLs with P-value < 0.05 and $\max (-\log P)>4$. (A-D) are the parameters derived from the 4-Parameter Logistic Model, and are provided in Table $\mathbf{1 .}$
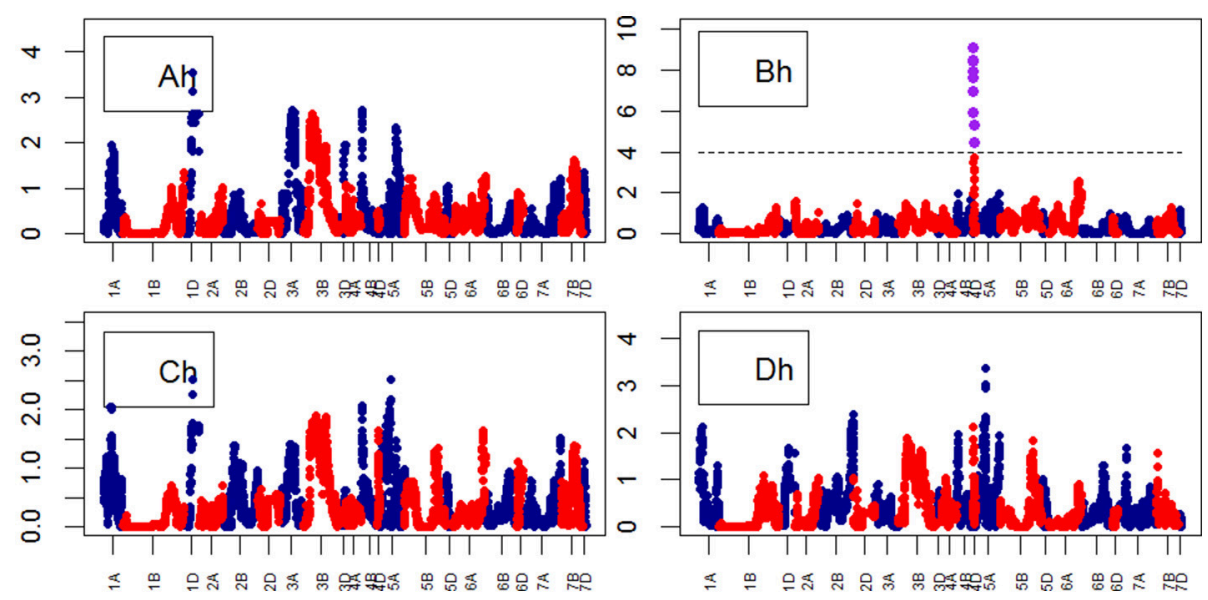

FIGURE 9 | Manhattan plots from the genetic mapping of the curve's growth parameters corresponding to the trait Height. Purple dots indicate QTLs with P-value < 0.05 and $\max (-\log P)>4$. (Ah-Dh) are the parameters (see Table 1) derived from the 4-Parameter Logistic Model for Height, and are provided in Table 1.

To look further into the effect of key markers over trait development, we fitted a regression model of the trait against the marker, for each time point. Results are summarized in Figures S7-S11. Briefly, RAC875_rep_c105718_585 (likely to be the Rht-B1b gene) marker showed a allelic effect on area, height, senescence and negative on Water Use, indicating a positive correlation with the trait. The Kukri_c27309_590 marker (likely to be related to the $P p d-D 1$ gene) showed a positive effect on area, height, and water use, indicating a positive correlation with the trait. However, for Senescence, the marker effect was positive up until 136 DAS but its significance level was lower after that time point which coincided with the average plant initiating Senescence. The marker IAAV1650 (closely linked to the vernalization gene $V r n-A 1$ ) showed completely different patterns for each trait: for Area, the effect was positive; for height, the effect was negative; for senescence the effect became positive after the average plant started senescence; and for water use the effect became negative at around 158 DAS. The BS00087197_51 (on chr7B) showed a positive effect on area and water use and negative on height. However, for senescence, the marker effect was negative up until 136 DAS but its significance level was lower after that time point, this pattern was the opposite of that showed by the Kukri_c27309_590 marker. Regardless of the marker, this analysis together with QTL mapping seems to suggest that fundamental changes take place around 136 DAS.

Genetic mapping of growth curves parameters fitted to the same traits confirmed the identification of major QTLs such as those on chr2D, chr4D, and on chr7B. This result demonstrate the power of analyzing growth curves that carry all the information about the plant's development as opposed to the analysis of single time points. However, it also showed 


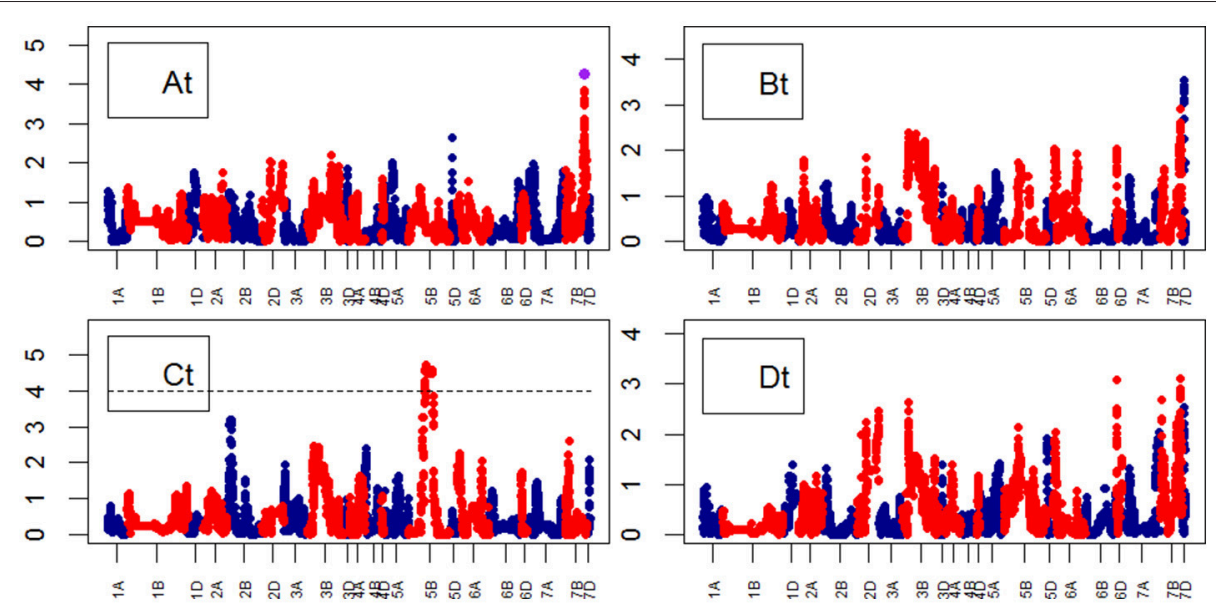

FIGURE 10 | Manhattan plots from the genetic mapping of the curve's growth parameters corresponding to the Senescence (TYP) Area. Purple dots indicate QTLS with $P$-value $<0.05$ and $\max (-\log P)>4$. (At-Dt) are the parameters (see Table 1) derived from the 4-Parameter Logistic Model for TYP (Senescence), and are provided in Table 1.
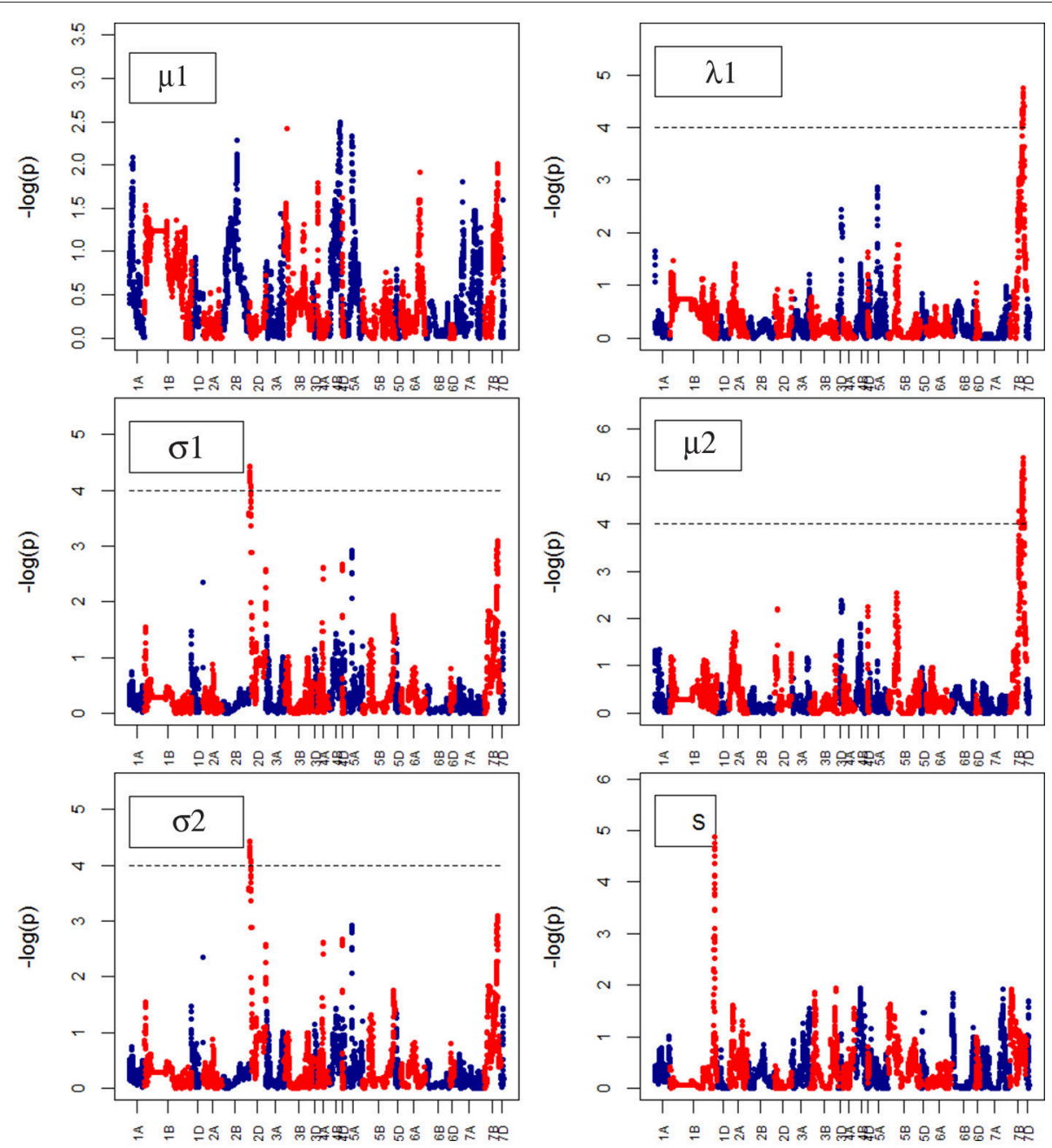

FIGURE 11 | Manhattan plots from the genetic mapping of the curve's growth parameters corresponding to Water Use. $\mu 1, \lambda 1, \sigma 1, \sigma 2$ and S are the parameters (see Table 1) derived from the (Polynominal logistic Model or Finite Mixture Model), and are provided in Table 1. 


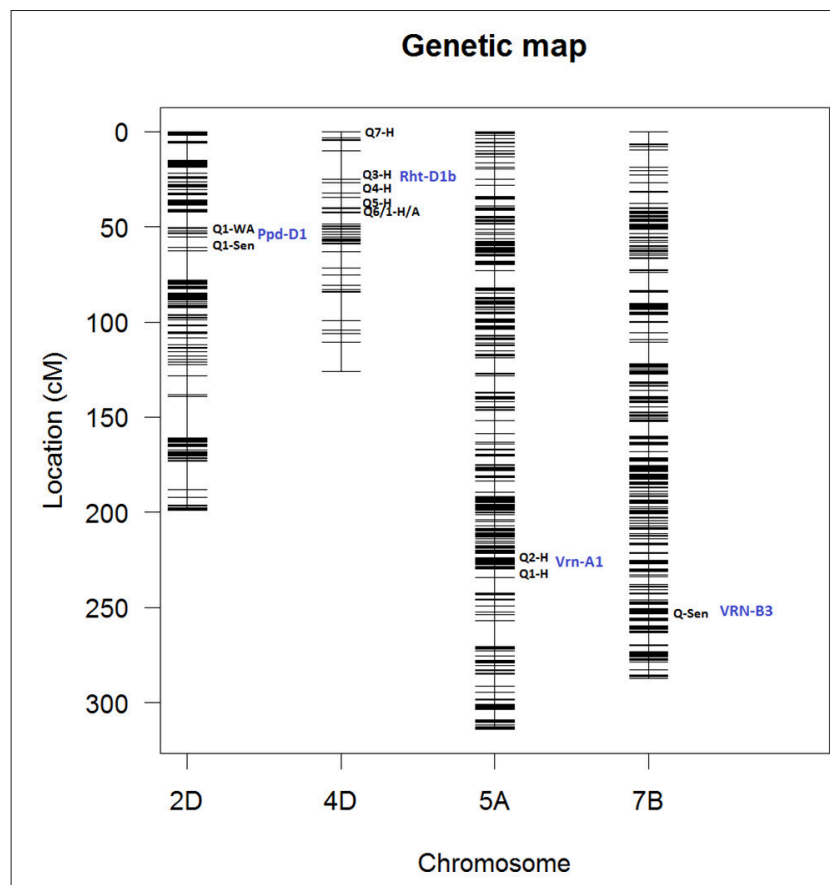

FIGURE 12 | Genetic map showing QTL locations for chromosomes 2D, corresponding to markers: (Q1) GENE_0137_147 and (Q1)

Kukri_c27309_590; 4D corresponding to markers: (Q7) Tdurum_contig420, (Q3) Kukri_rep_c68594_530, (Q4) RAC875_c1673_193, (Q5)

RAC875_c6922_291, (Q6/Q1) RAC875_rep_c105718_585; 5A, markers: (Q2) IAAV1650 and (Q1) BS00011360_51 and 7B, markers: BS00087197_51. Blue labels indicate the names associated to each chromosome location. Q\# indicate QTL number in Table 2. H, Height; TYArea, Senescence; A, Area; WA, Water Amount. More information about these QTLs is given in Table 2.

that the analysis of single time points helps to describe how traits and their marker effects evolve over time. Figure S11 shows a comparison of phenotypes vs. haplotypes of a number of RILS who carried different allelic variation for the markers $\mathrm{m} 1$ = RAC875_rep_c105718_585, m2 = BS00011360_51, m3 = IAAV1650 and m4 = Kukri_c27309_590. Although these four large QTL have been detected in this experiment and

\section{REFERENCES}

Al-Tamimi, N., Brien, C., Oakey, H., Berger, B., Saade, S., Ho, Y. S., et al. (2016). Salinity tolerance loci revealed in rice using high-throughput non-invasive phenotyping. Nat. Commun. 7:13342. doi: 10.1038/ncomms 13342

Anderegg, W. R. (2015). Spatial and temporal variation in plant hydraulic traits and their relevance for climate change impacts on vegetation. New Phytol. 205, 1008-1014. doi: 10.1111/nph.12907

Bentley, A. R., Scutari, M., Gosman, N., Faure, S., Bedford, F., Howell, P., et al. (2014). Applying association mapping and genomic selection to the dissection of key traits in elite european wheat. Theor. Appl. Genet. 127, 2619-2633. doi: 10.1007/s00122-014-2403-y

Bentley, A. R., Horsnell, R., Werner, C. P., Turner, A. S., Rose, G. A., Bedard, C., et al. (2013). Short, natural, and extended photoperiod response in bc2f4 lines of bread wheat with different photoperiod-1 (ppd-1) alleles. J. Exp. Bot. 64, 1783-1793. doi: 10.1093/jxb/ert038 they are known important controllers of plant growth and architecture in wheat (Vrn-A1, Rht-D1, and $P p d-D 1)$, there are no striking differences between these 16 plants representing all possible combination. This demonstrates the merit of accurate measurement taken here to quantify the parameters responsible for plant form and development.

This study used a new data driven approach to analyse the longitudinal data capturing the process of formation of dynamic traits such as senescence or water use. We combined the mapping power and precision of a MAGIC wheat population with robust computational methods to track the spatio-temporal dynamics of traits associated with wheat performance. We demonstrated that the combination of these two methodologies can be used as a powerful strategy for fine-tuning wheat's response to the environment.

\section{AUTHOR CONTRIBUTIONS}

$\mathrm{AC}$ and IM conceived analysis. $\mathrm{AB}$ and JD developed and obtained funding for the project. $\mathrm{JH}$ processed digital images. AC analyzed data. RM, IM, and AC assisted with the analysis. $\mathrm{KA}, \mathrm{FC}$, and KW provided technical support in the collection of phenotypic data. All authors provided comments and corrected the manuscript.

\section{ACKNOWLEDGMENTS}

Access to the Plant Phenomics Centre was provided by the Capability for Crop Phenotyping grant (BBSRC ref number $\mathrm{BB} / \mathrm{J004464/1}$ to JD). The creation of the NIAB MAGIC population was supported by $\mathrm{BB} / \mathrm{E} 007201 / 1$. We are grateful to the team of the Plant Phenomics Centre for carrying out the experiments. $\mathrm{AB}$ is supported by Designing Future Wheat $\mathrm{BB} / \mathrm{PO} 16855 / 1$.

\section{SUPPLEMENTARY MATERIAL}

The Supplementary Material for this article can be found online at: https://www.frontiersin.org/articles/10.3389/fpls.2018. 00887/full\#supplementary-material
Bresson, J., Vasseur, F., Dauzat, M., Koch, G., Granier, C., and Vile, D. (2015). Quantifying spatial heterogeneity of chlorophyll fluorescence during plant growth and in response to water stress. Plant Methods 11:23. doi: 10.1186/s13007-015-0067-5

Camargo, A., Azuaje, F., Wang, H., and Zheng, H. (2008). Permutation - based statistical tests for multiple hypotheses. Source Code Biol. Med. 3, 15-15. doi: 10.1186/1751-0473-3-15

Camargo, A. V., Mott, R., Gardner, K. A., Mackay, I. J., Corke, F., Doonan, J. H., et al. (2016). Determining phenological patterns associated with the onset of senescence in a wheat magic mapping population. Front. Plant Sci. 7:1540. doi: 10.3389/fpls.2016.01540

Ford, J. H., Patterson, T. A., and Bravington, M. V. (2015). Modelling latent individual heterogeneity in mark-recapture data with Dirichlet process priors. ArXiv.

Gimenez, O., Cam, E., and Gaillard, J.-M. (2017). Individual heterogeneity and capture-recapture models: what, why and how? Oikos 127, 664-686. doi: 10.1111/oik.04532 
Hathaway, R. J. (1985). A constrained formulation of maximumlikelihood estimation for normal mixture distributions. Ann. Statist. 13, 795-800.

Kiss, T., Balla, K., Veisz, O., Láng, L., Bed,o, Z., Griffiths, S., et al. (2014). Allele frequencies in the vrn-a1, vrn-b1 and vrn-d1 vernalization response and ppdb1 and ppd-d1 photoperiod sensitivity genes, and their effects on heading in a diverse set of wheat cultivars (Triticum aestivum 1.). Mol. Breed. 34, 297-310. doi: 10.1007/s11032-014-0034-2

Li, Z., and Sillanpää, M. J. (2013). A bayesian nonparametric approach for mapping dynamic quantitative traits. Genetics 194, 997-1016. doi: 10.1534/genetics.113.152736

Mackay, I. J., Bansept-Basler, P., Barber, T., Bentley, A. R., Cockram, J., Gosman, N. et al. (2014). An eight-parent multiparent advanced generation inter-cross population for winter-sown wheat: creation, properties, and validation. G3 4, 1603-1610. doi: 10.1534/g3.114. 012963

Mott, R., Talbot, C. J., Turri, M. G., Collins, A. C., and Flint, J. (2000). A method for fine mapping quantitative trait loci in outbred animal stocks. Proc. Natl. Acad. Sci. U.S.A. 97, 12649-12654. doi: 10.1073/pnas.2303 04397

Paine, C. E. T., Marthews, T. R., Vogt, D. R., Purves, D., Rees, M., Hector, A., et al. (2012). How to fit nonlinear plant growth models and calculate growth rates: an update for ecologists. Methods Ecol. Evol. 3, 245-256. doi: 10.1111/j.2041-210X.2011.00155.x

Poorter, H. (1989). Plant growth analysis: towards a synthesis of the classical and the functional approach. Physiol. Plant. 75, 237-244.

Soudzilovskaia, N. A., Elumeeva, T. G., Onipchenko, V. G., Shidakov, I. I., Salpagarova, F. S., Khubiev, A. B., et al. (2013). Functional traits predict relationship between plant abundance dynamic and longterm climate warming. Proc. Natl. Acad. Sci. U.S.A. 110, 18180-18184. doi: $10.1073 /$ pnas. 1310700110
Storey, J. D., and Tibshirani, R. (2003). Statistical significance for genomewide studies. Proc. Natl. Acad. Sci. U.S.A. 100, 9440-9445. doi: 10.1073/pnas.1530509100

Wu, C., Li, G., Zhu, J., and Cui, Y. (2011). Functional mapping of dynamic traits with robust t-distribution. PLoS ONE 6:e24902. doi: 10.1371/journal.pone.0024902

Xie, Q., Mayes, S., and Sparkes, D. L. (2015). Carpel size, grain filling, and morphology determine individual grain weight in wheat. J. Exp. Bot. 66, 6715-6730. doi: 10.1093/jxb/erv378

Xiong, H., Goulding, E. H., Carlson, E. J., Tecott, L. H., McCulloch, C. E., and Sen, S. (2011). A flexible estimating equations approach for mapping function-valued traits. Genetics 189, 305-316. doi: 10.1534/genetics.111.129221

Yang, R., Tian, Q., and Xu, S. (2006). Mapping quantitative trait loci for longitudinal traits in line crosses. Genetics 173, 2339-2356. doi: 10.1534/genetics.105.054775

Yang, R., and Xu, S. (2007). Bayesian shrinkage analysis of quantitative trait loci for dynamic traits. Genetics 176, 1169-1185. doi: 10.1534/genetics.106.064279

Zhang, C., Mapes, B. E., and Soden, B. J. (2003). Bimodality in tropical water vapour. Quar. J. R. Meteorol. Soc. 129, 2847-2866. doi: 10.1256/qj.02.166

Conflict of Interest Statement: The authors declare that the research was conducted in the absence of any commercial or financial relationships that could be construed as a potential conflict of interest.

Copyright (C) 2018 Camargo, Mackay, Mott, Han, Doonan, Askew, Corke, Williams and Bentley. This is an open-access article distributed under the terms of the Creative Commons Attribution License (CC BY). The use, distribution or reproduction in other forums is permitted, provided the original author(s) and the copyright owner(s) are credited and that the original publication in this journal is cited, in accordance with accepted academic practice. No use, distribution or reproduction is permitted which does not comply with these terms. 